\title{
POWER ALLOCATION METHOD BASED ON THE CHANNEL STATISTICS FOR COMBINED POSITIONING AND COMMUNICATIONS OFDM SYSTEMS
}

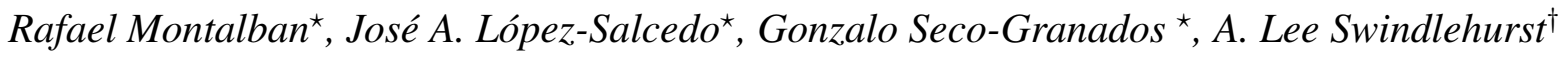 \\ * Universitat Autònoma de Barcelona, 08193 Bellaterra, SPAIN \\ \{rafael.montalban, jose.salcedo, gonzalo.seco\}@uab.cat \\ $\dagger$ University of California, Irvine, Irvine, CA 92697, USA \\ swindle@uci.edu
}

\begin{abstract}
The design of pilot and data power allocations for multicarrier OFDM signals is a key aspect in the development of combined positioning and high-data-rate communications systems. In this paper, we investigate capacity-maximizing pilot and data power allocations when a certain positioning accuracy is required. We consider a formulation based on the Expected Crámer-Rao Bound of the joint time-delay and channel estimation and the ergodic capacity, modeling the channel impulse response as a random vector. We compare the performance of capacity-maximizing pilot and data power distributions with respect to distributions that use equi-spaced and equi-powered pilot structures, shown by previous work to be optimal in terms of channel estimation. Numerical results show that the restriction to the use of equi-spaced and equi-powered pilot structures has an important impact on both the achievable capacity and the positioning capabilities of the designed signals.
\end{abstract}

Index Terms - OFDM, time-delay estimation, channel estimation, capacity, synchronization

\section{INTRODUCTION}

The use of multicarrier (MC) signals for combined positioning and high data rate communications systems requires accurate estimation of timing offset and channel impulse response in order to achieve desirable performance. As wireless MC systems rely on pilot symbols for channel estimation, it seems reasonable to apply these pilot symbols for time-delay estimation (TDE) as well (which is key for positioning). Both the pilot and the data power allocation need to be designed to obtain the best possible performance.

Optimal pilot design for channel estimation has been studied extensively [1-4], and results show that if equi-spaced and equipowered pilots are possible, then they are optimal in terms of channel estimation mean square error. Moreover, the problem of optimal power distribution between pilots and data was studied in [2]. Other work has studied the problem of achieving high TDE accuracy in MC systems. In [5,6], it was shown that for a pilot-only MC signal, minimizing the variance of the TDE requires maximizing the root-mean-square (or Gabor) bandwidth of the signal. In [7], the problem of pilot design is considered for combined time-delay and channel estimation in OFDM signals. In general, the optimal structures consist of pilot subcarriers at the edges of the bandwidth and approximately equi-spaced pilot subcarriers in between.

This work was partly supported by the Spanish Government under grant TEC2011-28219 and by the Catalan Government under grant 2009 SGR 298.
In $[8,9]$, we presented a nearly optimal power allocation approach as a solution to the problem of maximizing capacity in OFDM systems, while guaranteeing a certain level of TDE accuracy. The power allocation method presented was dependent on the knowledge of the specific channel impulse response, which makes sense for the design of uplink signals or when feedback is available.

This paper studies the problem of finding the capacity maximizing pilot and data distributions for OFDM signals, modeling the channel impulse response as a random vector. We focus on a scenario where a certain positioning capability is desired (i.e., we fix a desired TDE accuracy), and the system must be designed to achieve the highest possible capacity. We propose a method to design close-to-optimal pilot and data power allocations based on the channel statistics. The performance of the distributions obtained is compared to power allocations that use optimal pilot structures for channel estimation (i.e., equi-powered and equi-spaced in a circular sense). As our results show, restricting the pilot distributions to be equi-powered and equi-spaced produces a degradation on the maximum achievable capacity and TDE accuracy.

\section{SYSTEM MODEL AND PRELIMINARIES}

We use two performance metrics: a cost function based on the channel capacity when there is uncertainty in the channel state information, and the Expected Cramér-Rao Bound (ECRB) of the joint timedelay and channel estimates.

\subsection{OFDM Signal Model}

Consider the following frequency-selective channel model,

$$
h(t)=\sum_{l=0}^{L-1} h_{l} \delta\left(t-l T_{s}-\tau_{d}\right)
$$

where $L$ is an upper bound on the number of discrete multipath components, $h_{l}$ is the complex channel gain for the $l$-th path, $T_{s}$ is the sampling period and $\tau_{d}$ is the time-delay between source and receiver. Since the delay is explicitly modeled inside the terms $\delta\left(t-l T_{s}-\tau_{d}\right)$, the channel coefficients $\left\{h_{l}\right\}$ are independent of $\tau_{d}$. We define $\mathbf{h}=\left[h_{0}, h_{1}, \ldots, h_{L-1}\right]^{T}$, the vector containing the channel coefficients, as a random vector with $\mathbf{h} \sim \mathcal{C N}\left(\mathbf{0}, \boldsymbol{\Sigma}_{\mathbf{h}}\right)$. We consider the channel coefficients to be independent of each other (i.e., covariance matrix $\boldsymbol{\Sigma}_{\mathbf{h}}$ is diagonal).

We assume only the statistics of the channel are known, so both $\mathbf{h}$ and the channel delay $\tau_{d}$ need to be estimated through the use of pilot tones. For the scope of this paper, we restrict our problem to 
the zero inter-carrier and inter-symbol interference case. This means that the transmitter and the receiver are frequency synchronized, and that the duration of the cyclic prefix (CP) $T_{G}$ is larger than the delay spread plus the time-delay uncertainty (i.e. $\left.(L-1) T_{s}+\tau_{d}<T_{G}\right)$.

Consider now the vector containing the discrete Fourier transform (DFT) of $N$ samples of the received signal collected during an OFDM symbol:

$$
\mathbf{y}=\boldsymbol{\Gamma}\left(\tau_{d}\right) \mathbf{S}(\mathbf{s}) \mathbf{W}_{L} \mathbf{h}+\mathbf{n},
$$

where

$$
\begin{aligned}
\boldsymbol{\Gamma}\left(\tau_{d}\right)= & \operatorname{diag}\left(\left[e^{-j 2 \pi \frac{-(N-1) / 2}{T_{s}} \tau_{d}}, e^{-j 2 \pi \frac{-(N-1) / 2+1}{T_{S}} \tau_{d}}\right.\right. \\
& \left.\left.\ldots, e^{-j 2 \pi \frac{(N-1) / 2}{T_{s}} \tau_{d}}\right]\right) \\
\mathbf{s}= & {\left[s_{-(N-1) / 2}, s_{-(N-1) / 2+1}, \ldots, s_{(N-1) / 2}\right]^{T} } \\
\mathbf{S}(\mathbf{s})= & \operatorname{diag}(\mathbf{s}) .
\end{aligned}
$$

$\mathbf{W}_{L}$ is composed of the first $L$ columns of the zero-frequency centered $N \times N$ Fourier matrix, $\mathbf{n}$ is a vector of additive Gaussian noise and $\mathbf{s}$ contains the symbols being transmitted.

\subsection{The Expected Cramér-Rao Bound}

We define the following parameter vector,

$$
\boldsymbol{\Theta}:=\left[\tau_{d}, \Re\left\{\mathbf{h}^{T}\right\}, \Im\left\{\mathbf{h}^{T}\right\}\right] \in \mathbb{R}^{(2 L+1) \times 1},
$$

which contains the time-delay information and the real $\left(\Re\left\{\mathbf{h}^{T}\right\}\right)$ and imaginary $\left(\Im\left\{\mathbf{h}^{T}\right\}\right.$ ) parts of the channel response. The CRB of these parameters has been presented previously in [7]. Based on the known result, we can approximately compute the ECRB as $\mathbf{E C R B}=E_{h}\{\mathbf{C R B}\}$, and we obtain

$$
\mathbf{E C R B}_{\tau_{d}, \mathbf{h}} \approx \frac{\sigma_{n}^{2}}{2}\left[\begin{array}{cc}
\mathrm{ECRB}_{11} & \mathbf{0} \\
\mathbf{0} & \mathbf{E C R B}_{22}
\end{array}\right]
$$

where

$$
\begin{aligned}
& \operatorname{ECRB}_{11}^{-1} \approx \operatorname{trace}\left(\mathbf{W}_{L}^{H} \mathbf{P} \mathbf{D}^{2} \mathbf{F}_{L} \boldsymbol{\Sigma}_{\mathbf{h}}\right)_{-} \\
& -\operatorname{trace}\left(\mathbf{W}_{L}^{H} \mathbf{P D} \mathbf{W}_{\mathbf{L}}(\mathbf{Q})^{-1}\right. \\
& \left.\cdot \mathbf{W}_{L}^{H} \mathbf{D P} \mathbf{W}_{L} \boldsymbol{\Sigma}_{\mathbf{h}}\right) \\
& \mathbf{E C R B}_{22} \approx\left[\begin{array}{c}
\Re\left\{\mathbf{Q}^{-1}\right\}+\frac{\frac{1}{2} \Re\left\{E_{h}\left\{\mathbf{q q}^{H}\right\}\right\}}{\operatorname{ECRB}_{11}^{-1}} \\
\Im\left\{\mathbf{Q}^{-1}\right\}+\frac{\frac{1}{2} \Im\left\{E_{h}\left\{\mathbf{q q}^{H}\right\}\right\}}{\operatorname{ECRB}_{11}^{-1}}
\end{array}\right. \\
& -\Im\left\{\mathbf{Q}^{-1}\right\}-\frac{\frac{1}{2} \Im\left\{E_{h}\left\{\mathbf{q q}^{H}\right\}\right\}}{\mathrm{ECRB}_{11}^{-1}} \\
& \left.\Re\left\{\mathbf{Q}^{-1}\right\}+\frac{\frac{1}{2} \Re\left\{E_{h}\left\{\mathbf{q q}^{H}\right\}\right\}}{\mathrm{ECRB}_{11}^{-1}}\right] \\
& E_{h}\left\{\mathbf{q q}^{H}\right\}=\mathbf{Q}^{-1} \mathbf{W}_{L}^{H} \mathbf{P} \mathbf{D} \mathbf{W}_{L} \Sigma_{h} \\
& \cdot \mathbf{W}_{L}^{H} \mathbf{P D} \mathbf{W}_{L} \mathbf{Q}^{-1} \\
& \mathbf{Q}=\mathbf{W}_{L}^{H} \mathbf{P} \mathbf{W}_{L} \\
& \mathbf{D}=\frac{2 \pi}{T_{s}} \operatorname{diag}\left(\left[-\frac{N-1}{2}, \ldots, \frac{N-1}{2}\right]\right) \\
& \mathbf{P}=\mathbf{S}^{H} \mathbf{S}=\operatorname{diag}\left(\mathbf{p}_{p}\right) \text {. }
\end{aligned}
$$

It is important to note that in several steps of the derivation the Laplace approximation $E\left\{\frac{X}{Y}\right\} \approx \frac{E\{X\}}{E\{Y\}}$ has been used. For the derivation of (7), we have ignored the presence of data symbols for the purpose of estimation, and have assumed that the vector $\mathbf{s}$ only contains pilot symbols. Note that the ECRB only depends on the power of the pilot subcarriers and not on the specific pilot symbols allocated.

\subsection{Capacity}

In [10] and [11] a lower bound on the channel capacity for serial transmissions over flat-fading channels was derived. If we apply this lower bound on a per-subcarrier basis, and summing across the data subcarriers, we have the following lower bound on the MC signal channel capacity is

$$
C \geq C_{\mathrm{lb}}=\frac{1}{N} E_{h, \hat{g}_{i}}\left\{\sum_{i \in \Omega} \log \left(1+\mathrm{SNReq}_{i}\right)\right\},
$$

where $\Omega$ contains the indices of the subcarriers allocated to data transmission, and where an equivalent signal-to-noise ratio for subcarrier $i$ is defined as,

$$
\mathrm{SNReq}_{i}=\frac{\left|\hat{g}_{i}\right|^{2} p_{d, i}}{\sigma_{g, i}^{2} p_{d, i}+\sigma_{n}^{2}},
$$

where $\hat{g}_{i}$ is the channel frequency response estimate for subcarrier $i$, $p_{d, i}$ is the power allocated to data transmission in subcarrier $i, \sigma_{g, i}^{2}$ is the variance of the channel response estimate for that subcarrier and $\sigma_{n}^{2}$ is the Gaussian noise power. The inequality in (14) becomes an equality only in the worst case, in which channel estimation uncertainty has the worst possible effect [10].

For an unbiased estimator, the value of $\sigma_{g, i}^{2}$ is lower bounded by the corresponding Crámer Rao Bound. This CRB can be can be easily computed as a function of parameters from the CRB of the channel impulse response estimate by taking into account that $\hat{\mathrm{g}}=\mathbf{W}_{L} \hat{\mathbf{h}}$.

In this paper, we consider the maximization of a cost function that approximates the lower bound on the capacity assuming that the lowest possible uncertainty in the channel estimates is achieved. Using the approximation $E\{\log (x)\} \lesssim \log (E\{x\})$, we can write

$$
C^{\prime} \doteq \frac{1}{N} \sum_{i \in \Omega} \log \left(1+E_{h, \hat{g}_{i}}\left\{\mathrm{SNReq}_{i}\right\}\right) \gtrsim C_{\mathrm{lb}}
$$

and applying the Laplace approximation we have that,

$$
C^{\prime} \approx \frac{1}{N} \sum_{i \in \Omega} \log \left(1+\frac{p_{d, i} \operatorname{tr}\left\{\mathbf{w}_{L, i}^{H} \mathbf{w}_{L, i}\left(\boldsymbol{\Sigma}_{h}+\mathbf{E C R B}_{22}\right)\right\}}{p_{d, i} \mathrm{ECRBg}_{i}+\sigma_{n}^{2}}\right),
$$

where $\mathbf{w}_{L, i}$ is row $i$ of matrix $\mathbf{W}_{L}$. As a result of the approximations used, our cost function is no longer a lower bound on the capacity but an approximation of it.

\section{POWER ALLOCATION OPTIMIZATION}

\subsection{Arbitrary Pilot Distributions}

In order to maximize our cost function (17) while ensuring a certain level of TDE accuracy, we need to adequately distribute pilot and data power across the subcarriers. 
One possible formulation of the problem is

$$
\left\{\begin{array}{cl}
\underset{\mathbf{p}, \mathbf{b}}{\max } & C^{\prime}(\mathbf{p}, \mathbf{b}) \\
\text { s.t. } & \operatorname{ECRB}_{11}(\mathbf{p}, \mathbf{b}) \leq \beta \\
& \mathbf{p}^{T} \cdot \mathbf{1} \leq P_{T} \\
& \mathbf{p} \geq \mathbf{0} \\
& b_{i}^{2}-b_{i}=0
\end{array}\right.
$$

where vector $\mathbf{p}$ contains the powers assigned to each of the subcarriers, $\mathbf{b}$ is a binary vector of length $N$ that contains a ' 1 ' in the positions corresponding to pilot subcarriers and a ' 0 ' in the positions corresponding to data subcarriers, and $P_{T}$ is the total available power. Thus, the elements of vector $\mathbf{p}_{p}$ containing the pilot powers can be expressed as $p_{p, i}=b_{i} p_{i}$ and the elements of vector $\mathbf{p}_{d}$ containing data powers as $p_{p, i}=p_{i}(1-b i)$ (i.e., $\left.\mathbf{p}=\mathbf{p}_{p}+\mathbf{p}_{d}\right)$.

While the first constraint in (18) ensures that the ECRB of the time-delay estimate is lower than a certain value $\beta$, the fourth restriction in (18) causes the problem to be combinatorial, i.e., the subcarriers are going to be assigned to either pilot or data symbols. However, the problem can be relaxed to eliminate its combinatorial constraint.

If we eliminate the fourth restriction in (18), the subcarriers can be shared by data and pilot symbols simultaneously. The solution to this relaxed problem can be found numerically using standard convex programming, which is guaranteed to converge to an optimal solution. Note that an analytical prove of the convexity of the cost function and the constraints in (18) is still a work in progress, but extensive simulation shows the optimization problem is convex.

Simulation results show that the number of subcarriers that contain pilot power is very low, generally equal or close to $L+1$, which is the lowest value required for the FIM to be full rank. In terms of our formulation, we see that optimal solutions for the relaxed problem consist of a vector $\mathbf{b}$ with most of its entries set to ' 0 ' and just a few are set to values greater than ' 0 ', which agrees with the results obtained in [7]. This almost binary $\mathbf{b}$ could then be approximated by a completely binary assignment and used to easily solve (18). The resulting power distribution is close or equal to the global optimum.

\subsection{Equi-spaced and Equi-powered Pilot Distributions}

We consider now the case where we restrict ourselves to use equipowered and equi-spaced pilot distributions only. For the sake of brevity, we will refer to this kind of pilot distributions as "comb" distributions in the sequel.

We consider a pilot distribution that uses $K$ out of the $N$ tones in an OFDM symbol. In order for the pilots to be equi-spaced, $N$ must be a multiple of $K$, i.e. $N=K J$, for some integer $J$. The set of positions of an equi-spaced distribution is $\mathcal{I}=\{j+J k \mid k \in$ $[0, K-1]\}$ for some $j \in[0, J-1]$. Pilot subcarriers are equipowered in this case, so each subcarrier will receive a fraction of the total power devoted to pilot transmission $P_{p}$. In this case, the vector containing the pilot powers $\mathbf{p}_{p}$ is just a function of $j$ and $P_{p}$.

Note that $K$ must be at least $L+1$ for the estimation problem to be feasible. Ideally, we would like to use the minimum number of pilots, i.e. $K=L+1$, in order to have as many subcarriers free for data transmission as possible. However, depending on the values of $N$ and $L$, this may be impossible. In the sequel, we assume that $N$ is a multiple of $L+1$ to focus our analysis on the effect of employing comb pilot distributions.

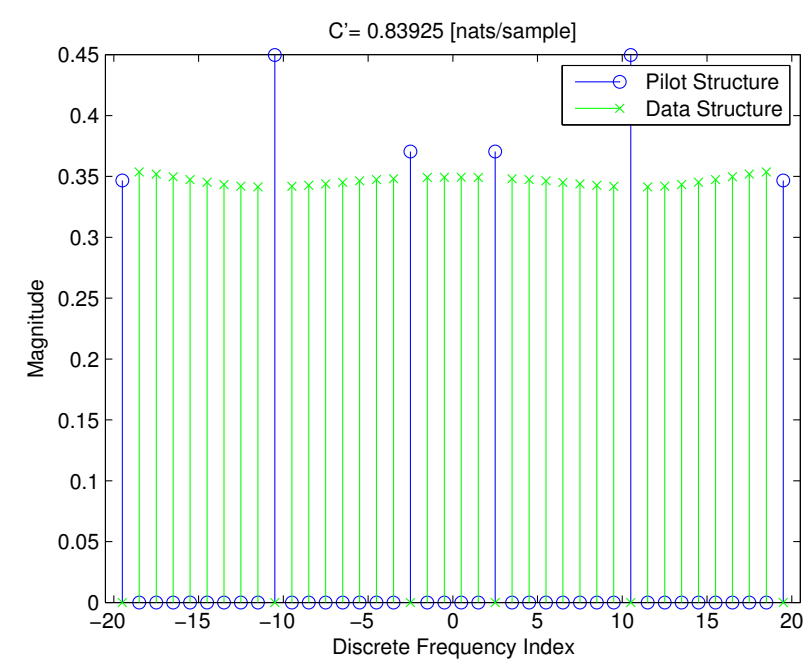

Fig. 1. Example pilot and data power distribution using arbitrary pilot allocation.

The maximization problem we consider is:

$$
\begin{cases}\max _{P_{p}, \mathbf{p}_{d}, j} & C^{\prime}\left(\mathbf{p}_{p}\left(P_{p}, j\right), \mathbf{p}_{d}\right) \\ \text { s.t. } & \operatorname{ECRB}_{11}\left(\mathbf{p}_{p}\left(P_{p}, j\right)\right) \leq \beta \\ & P_{p}+\mathbf{p}_{d}^{T} \cdot \mathbf{1} \leq P_{T} \\ & \mathbf{p}_{p}^{T} \cdot \mathbf{p}_{d}=0 \\ & \mathbf{p}_{d} \geq \mathbf{0}\end{cases}
$$

where the pilot distribution $\mathbf{p}_{p}$ is forced to be a comb distribution, and thus is a function of $P_{p}$ and $p_{d}$. This problem can be easily solved numerically. Note that the most favorable shift of the comb distribution is selected in the simulations since $j$ is one of the optimization variables.

It is also interesting to define a new problem that will be used in the next section in order to gain a deeper insight on how the capacity and TDE accuracy change with respect to those achieved by an arbitrary pilot power distribution that devotes the same amount of power to pilot and data transmission. The problem is obtained from (19) by eliminating the $\mathrm{ECRB}_{11}$ constraint and fixing a value of $P_{p}$. In this case, the goal is to maximize capacity for the pilot power without consideration of TDE accuracy.

\section{PERFORMANCE ANALYSIS}

In this section we study the performance of several pilot and data power distributions obtained as solutions to the optimization problems discussed in Section 3. For the results presented, we assume a channel impulse response of length $L=4$ and an OFDM signal of $N=40$ subcarriers. The total power for pilots plus data has been fixed to $P_{T}=5$ and the noise variance to $\sigma_{n}^{2}=10^{-2}$. The maximum acceptable value of $\mathrm{ECRB}_{11}$ is constrained to be $\beta=0.002$ and a diagonal channel covariance matrix has been used $\left(\boldsymbol{\Sigma}_{h}=\operatorname{diag}([1.8949,1.6222,1.4209,1.9405])\right)$. 


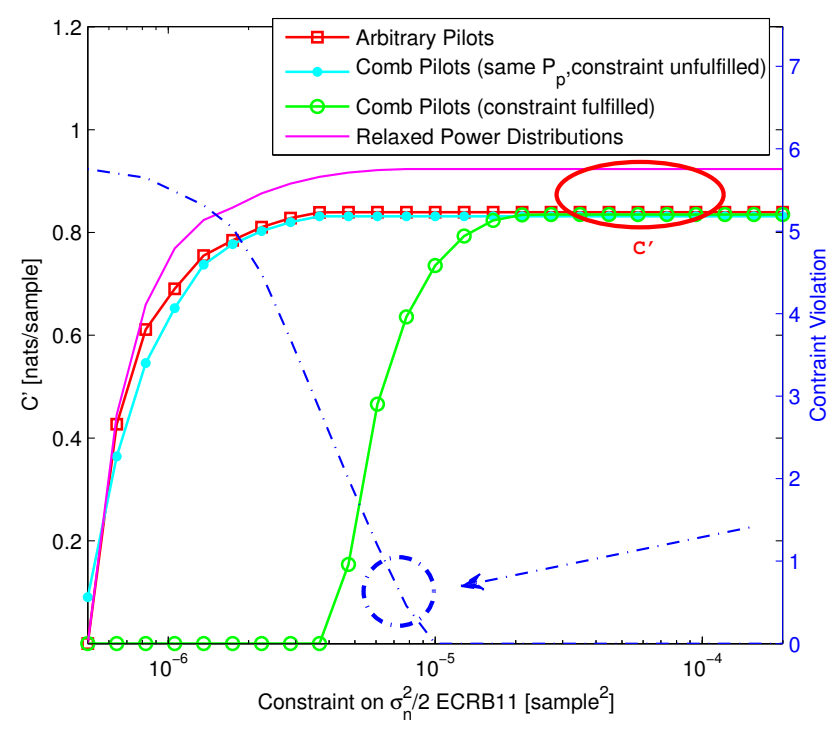

Fig. 2. Channel capacity versus TDE accuracy.

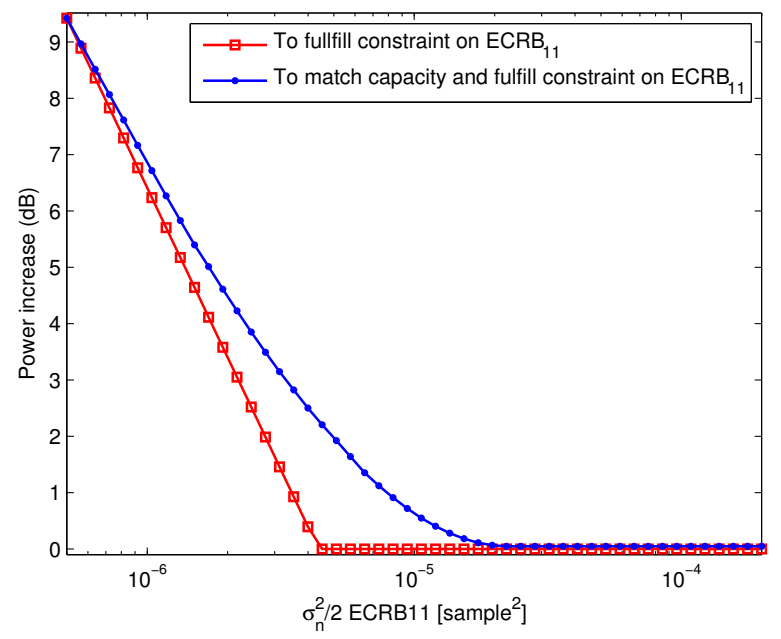

Fig. 3. Increase in total power required by comb pilot distributions to match the performance of an arbitrary distribution.

Figure 1 shows a solution to (18) designed to maximize $C^{\prime}$ with a constraint on the maximum $\mathrm{ECRB}_{11}$. As our channel model assumes the channel coefficients are independent of each other, $\boldsymbol{\Sigma}_{h}$ is a diagonal matrix and $E\left\{\left|\hat{g}_{i}\right|^{2}\right\}$ is the same for all $i$. This results in a practically flat data power distribution and a somewhat equispaced pilot distribution, with the two subcarriers at the edge of the bandwidth occupied by pilot symbols. For the same parameter values, solutions to (19) show mostly flat data power distribution, but for restrictive values of $\beta$ a substantial amount of extra pilot power is required to fulfill the constraint on $\mathrm{ECRB}_{11}$, which impacts the achievable capacity. For the example presented, there is a reduction of the $12.4 \%$ in capacity caused by the use of comb pilot allocations.

Figure 2 studies the trade-off between capacity and TDE uncertainty. The curve with square markers represents the capacity achieved by distributions obtained through the maximization of $C^{\prime}$ without any restriction on the pilot distributions that may be used, i.e. solutions to (18). The curve with circle markers corresponds to distributions designed with comb pilot structures that fulfill the constraint on TDE accuracy, i.e. solutions to (19). Note that in any case where the total available power is not enough for a comb pilot structure to fulfill the constraint, the capacity is 0 since all power is assigned to pilot transmission. The curve with point markers corresponds to power allocations that use comb pilot distributions with the same total power for pilot transmission as in the arbitrary-pilots case. Note that in this case the distributions do not achieve the desired ECRB and the amount they violate the constraint is represented by the dash-dot curve. The performance of solutions to the relaxed version of (18) is provided as a reference of the maximum achievable capacity (solid line without markers).

Figure 3 shows the increase in total power required by a distribution with comb pilot structure to match the performance of a distribution with arbitrary pilots. The line with square markers represents the extra power needed to achieve the same TDE accuracy (i.e. the same $\mathrm{ECRB}_{11}$ ), while the curve with point markers represents the additional power needed to achieve the same capacity and positioning accuracy.

Results show that for a fixed amount of power devoted to pilot transmission, a distribution with comb pilots obtains similar results in terms of capacity as an arbitrary distribution that has to fulfill a strict constraint on the TDE accuracy. This means that comb pilots are adequate when one is only interested in maximizing capacity (i.e., designing a communications system), which agrees with previous results in the literature. However, it is also clear from our results that comb pilot distributions are inadequate when the positioning capabilities of the signal are a concern. In order to match the performance of an arbitrary distribution, comb distributions require much higher amounts of power.

\section{CONCLUSIONS}

In this paper, we have considered the problem of designing pilot and data power distributions with close-to-optimal performance in terms of capacity and positioning capabilities when only statistical knowledge of the channel is available. We have studied the performance of near-optimal pilot and data power allocations in comparison to the traditional solution of using equi-spaced and equi-powered pilot distributions. Results show that equi-spaced and equi-powered pilot structures are not adequate for TDE as they require important increases in the amount of power devoted to pilot transmission to achieve the same TDE accuracy, which results in decreased capacities. However, when the same amount of power is devoted to pilot transmission, equi-spaced and equi-powered distributions can achieve similar performance in terms of capacity compared to arbitrary distributions. Thus, equi-spaced and equi-powered pilot distributions are only a good choice when designing a system solely for communication purposes.

\section{REFERENCES}

[1] S. Ohno and G.B. Giannakis, "Optimal training and redundant precoding for block transmissions with application to wireless OFDM," IEEE Transactions on Communications, vol. 50, no. 12, pp. 2113 - 2123, dec 2002.

[2] S. Ohno and G.B. Giannakis, "Capacity maximizing MMSEoptimal pilots for wireless OFDM over frequency-selective 
block rayleigh-fading channels," IEEE Transactions on Information Theory, vol. 50, no. 9, pp. 2138 - 2145, sept. 2004.

[3] R. Negi and J. Cioffi, "Pilot tone selection for channel estimation in a mobile OFDM system," Consumer Electronics, IEEE Transactions on, vol. 44, no. 3, pp. 1122-1128, 1998.

[4] I. Barhumi, G. Leus, and M. Moonen, "Optimal training design for MIMO OFDM systems in mobile wireless channels," Signal Processing, IEEE Transactions on, vol. 51, no. 6, pp. 1615-1624, 2003.

[5] F. Zanier and M. Luise, "Fundamental issues in time-delay estimation of multicarrier signals with applications to nextgeneration GNSS," in 10th International Workshop on Signal Processing for Space Communications SPSC 2008, 2008, pp. $1-8$.

[6] F. Zanier and M. Luise, "A new look into the issue of the Cramér-Rao bound for delay estimation of digitally modulated signals," in Proceedings of the 2009 IEEE International Conference on Acoustics, Speech and Signal Processing, 2009, pp. 3313-3316.

[7] M.D. Larsen, G. Seco-Granados, and A.L. Swindlehurst, "Pilot optimization for time-delay and channel estimation in OFDM systems," in Acoustics, Speech and Signal Processing (ICASSP), 2011 IEEE International Conference on. IEEE, 2011, pp. 3564-3567.

[8] R. Montalban, G. Seco-Granados, and A.L. Swindlehurst, "Suboptimal method for pilot and data power allocation in combined positioning and communications OFDM systems," in 2012 Asilomar Conference on Signals, Systems, and Computers, 2012.

[9] R. Montalban, J.A. López-Salcedo, G. Seco-Granados, and A.L. Swindlehurst, "Power allocation approaches for combined positioning and communications OFDM systems," in 2013 IEEE 14th Workshop on Signal Processing Advances in Wireless Communications (SPAWC) (in review), 2013.

[10] M. Medard, "The effect upon channel capacity in wireless communications of perfect and imperfect knowledge of the channel," Information Theory, IEEE Transactions on, vol. 46, no. 3, pp. 933-946, 2000.

[11] E. Biglieri, J. Proakis, and S. Shamai, "Fading channels: Information-theoretic and communications aspects," Information Theory, IEEE Transactions on, vol. 44, no. 6, pp. 26192692, 1998. 